\title{
AN IMPROVED OPERATING MODE FOR A Si(Li) X-RAY SPECTROMETER
}

N. W. Madden, F. S. Goulding, J. M. Jaklevic,

D. A. Landis and C. S. Rossington

Engineering Division

Lawrence Berkeley Laboratory

University of California

1 Cyclotron Rd.

Berkeley, CA 94720

This work was supported by the Director, Office of Energy Research, Office of Health and Environmental Research, Physical and Technological Division of the U.S. Department of Energy under Contract No. DE-AC03-76SF00098.

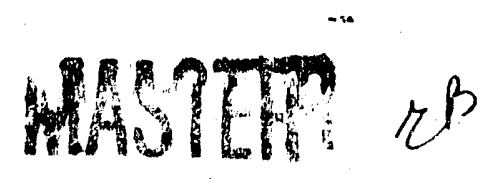




\title{
AN IMPROVED OPERATING MODE FOR A $\mathrm{Si}(\mathrm{Li}) \mathrm{X}$-RAY SPECTROMETER
}

\author{
N. W. Madden, F. S. Goulding, J. M. Jaklevic, \\ D. A. Landis and C. S. Rossington \\ Engineering Division \\ Lawrence Berkeley Laboratory \\ 1 Cyclotron Rd. \\ Berkeley, CA 94720
}

\begin{abstract}
A new technique has been developed for restoring the charge to the feedback capacitor in charge sensitive preamplifiers. The method, termed "transient-reset," was developed for use in very low noise $\mathrm{Si}(\mathrm{Li}) \mathrm{X}$-ray spectrometers. It has proved valuable as a diagnostic tool for evaluating the noise components of the input circuit in these systems. The technique is described and compared with the pulsedlight and transistor-reset methods. To complement the charge restoration technique, a new configuration of $\mathrm{Si}(\mathrm{Li})$ $\mathrm{X}$-ray detector with a very thin aluminum entrance window has been developed. Measurements of the effective window thickness are compared with similar results obtained on gold, nickel, and ion-implanted boron contacts.
\end{abstract}

\section{SCIENTIFIC MOTIVATION}

The problem of "dead" layers at the entry window of $\mathrm{Si}(\mathrm{Li}) \mathrm{X}$-ray detectors is common to ill low-energy $\mathrm{X}$-ray applications of these detectors. One such application is to grazing angle $\mathrm{X}$-ray telescopes that have made possible observations of weak stellar $X$-ray sources. The focal plane detectors used in many of these telesropes are gas proportional flow counters and $\mathrm{Si}(\mathrm{Li}) \mathrm{X}$-ray detectors. Gas pror.orional counters can be made with large areas but have only moderate energy resolution at low energies. They are used frequently with short foca! length telescopes. Newer telescopes, such as that designed for the broad band X-ray telescope "BBXRT" project at Goddard Space Flight Center, have small focal spots $(4-5 \mathrm{~mm})$ which match $\mathrm{Si}(\mathrm{Li}) \mathrm{X}$ ray detectors that offer excellent energy resolution. Since these instruments operate in the vacuum of space it is possible to observe low-energy $\mathrm{X}$-rays such as those from carbon, boron and beryllium if the spectrometer has suitably low noise and very thin entrance windows. The work described here was aimed at these applications.

\section{NOISE LIMITATIONS}

Careful analysis of the noise characteristics of very good $\mathrm{Si}(\mathrm{Li}) \mathrm{X}$-ray spectrometers shows that the limiting noise source is $1 / \mathrm{f}$ noise. For example, Fig. 1 is a noise plot of a

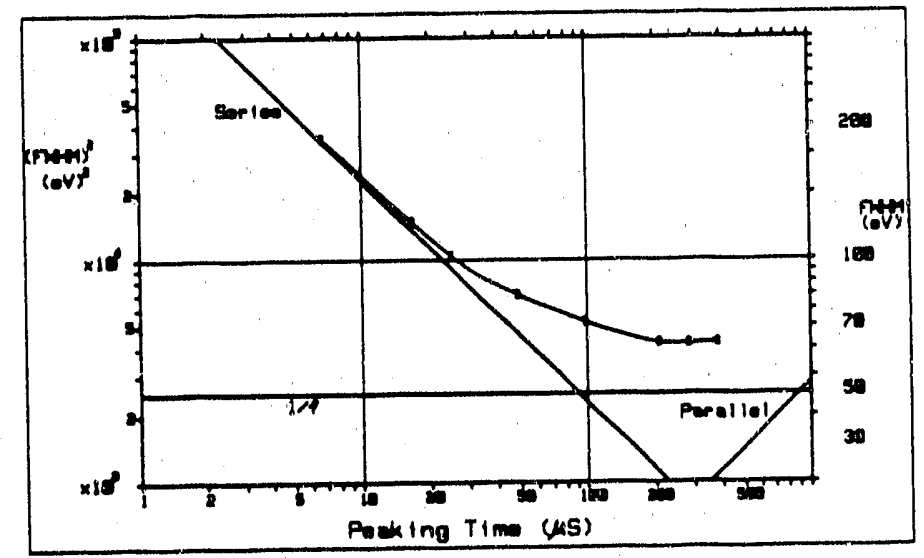

Fig. 1: Plot of FWHM resolution squared vs. peaking time of $\mathrm{Si}(\mathrm{Li}) \mathrm{X}$-ray spectrometer displaying the series, parallel and $1 / \mathrm{f}$ noise components.

spectrometer measured with a 6th order pseudo-Gaussian pulse shaper. The flat minimum in the noise plot shows the dominance of $1 / \mathrm{f}$ noise which limits the resolution of the system for X-ray energies less than $1 \mathrm{keV}$. Series $1 / \mathrm{f}$ noise is associated, at low operating temperatures, with trapping/detrapping at impurities and defects in the conducting channel of the FET. Parallel $1 / \mathrm{f}$ noise, on the other hand, is believed to originate in lossy dielectrics which are capacitively coupled into the FET gate, and possibly in surface layers of the detectors [1]. Careful control of impurities in the FET manufacturing process has reduced the magnitude of the series $1 / f$ noise. In some Interfet devices, the $1 / \mathrm{f}$ noise is less than the channel series noise at all frequencies down to $3 \mathrm{~Hz}$ [2]. One way to determine the presence of trapping/detrapping noise at sites in the FET channel is to perform noise measurements at different temperatures and frequencies in a grounded-gate configuration. Parallel input circuit noise is eliminated in this case. Figures $2 A$ and $2 B$ show such measurements of two small geometry $\mathrm{N}$-channel junction FETs [3]. In Fig. 2A, a Motorola device clearly exhibits bumps in the plot that accompany series $1 / \mathrm{f}$ noise. In Fig. 2B, a smaller device by Interfet exhibits higher 

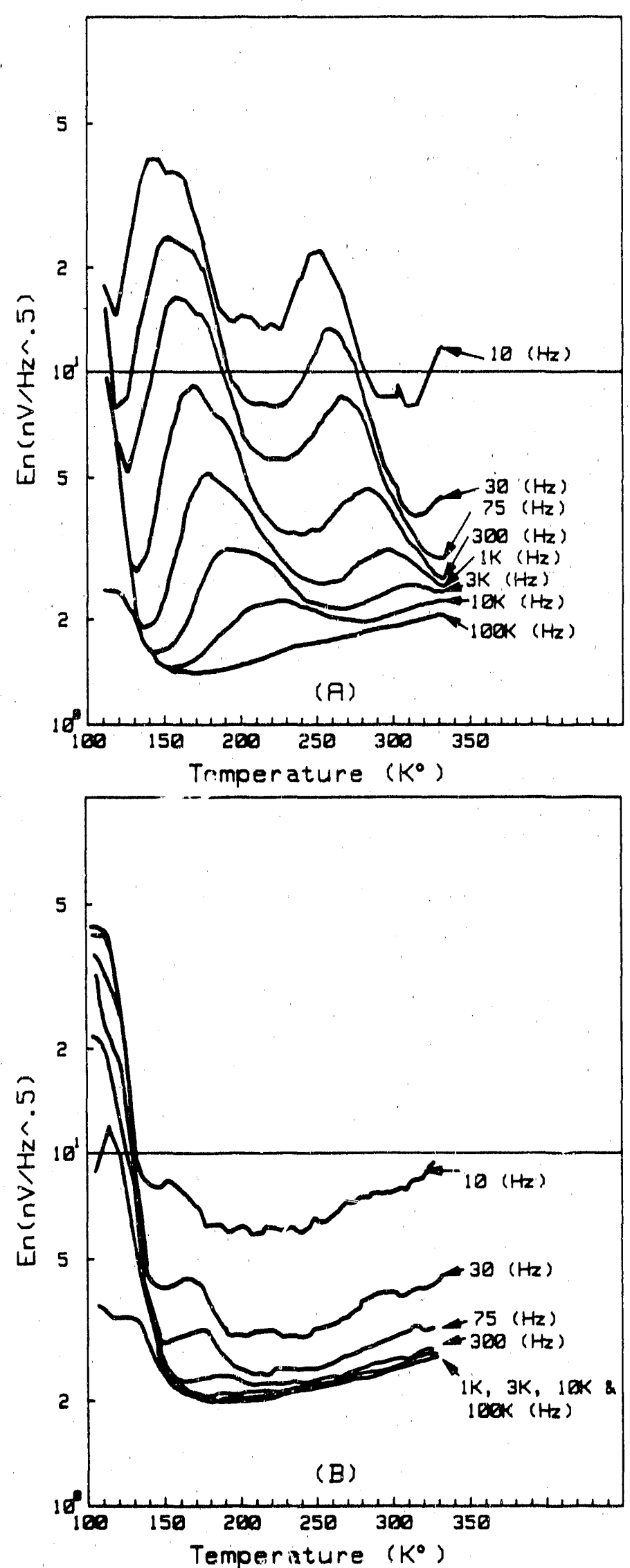

Fig. 2: Plot of channel noise as a function of temperature and frequency for A) Motorola 2N4416 FET and B) Interfet NJ14LOI. noise at the higher frequencies as expected due to the lower transconductance, but shows bumps only at very low frequencies indicating very little series $1 / \mathrm{f}$ noise. With regard to parallel $1 / \mathrm{f}$ noise, experience suggests that the highpurity boron nitride used to package the FET chip and its companion LED in a light-tight package is a major source of parallel $1 / \mathrm{f}$ noise. The new charge restoration technique avoids the use of a pulsed-light reset, and since the FET package need not be light-tight, dielectrics with much better loss characteristics than boron nitride can be used. The utilization of both lower loss dielectrics associated with the new charge restoration technique and modern selected FETs make it possible to largely eliminate both parallel and series $1 / \mathrm{f}$ noise.

\section{CHIARGE RESTORATION}

Virtually all modern low noise preamplifiers used in conjunction with $\mathrm{Si}(\mathrm{Li}) \mathrm{X}$-ray spectrometers employ a charge sensitive stage. Charge restoration is accomplished by either pulsed-light reset [4] or one of the several variants of transistor-reset [5]. Figure $3 \mathrm{~A}$ is a schematic representation of a pulsed-light reset preamplifier. The detector current flows from the virtual ground (gate of the FET) to the negative detector bias. If the preamplifier is operated without detector current flowing, it will immediately saturate, due to the FET drain-gate leakage, precluding any measurements of the FET. In normal operation, when the FET is operated with a reverse-biased detector, the companion LED will be turned on when the preamplifier output reaches a predetermined level. A large positive current $(n A-\mu A)$ then flows into the virtual ground, the result of illuminating the FETs drain-to-gate diode. As the charge is restored and the preamplifier output reaches a predetermined level, the LED is switched off and the cycle begins again. Pulsed-light reset was originally designed to minimize input circuit components. However, drawbacks include the inability to handle reverse-polarity detectors, the requirement of a light-tight package for the FET and LED, and the impairment of resolution at high rates due to residual aftereffects of the light [4].

A transistor-reset preamplifier is shown in Fig. 3B. It uses a selected low capacitance grounded-base bipolar transistor to feed the reset charge to the FET gate. Either polarity of charge can be restored by selection of the appropriate bipolar transistor. A light-tight package is not required and - very high count rates with little or no resolution degradation can be achieved [6]. However, the small additional capacitance $(0.3-0.5 \mathrm{pF})$ introduced in the gate circuit causes a significant degradation of resolution in low noise (low detector capacitance) spectrometers. This additional noise could be reduced by using a very low capacitance transistor specially fabricated for this application.

Figure $3 \mathrm{C}$ is a schematic representation of a transientreset preamplifier. Charge restoration is initiated when the preamplifier output reaches a predetermined lower level. 

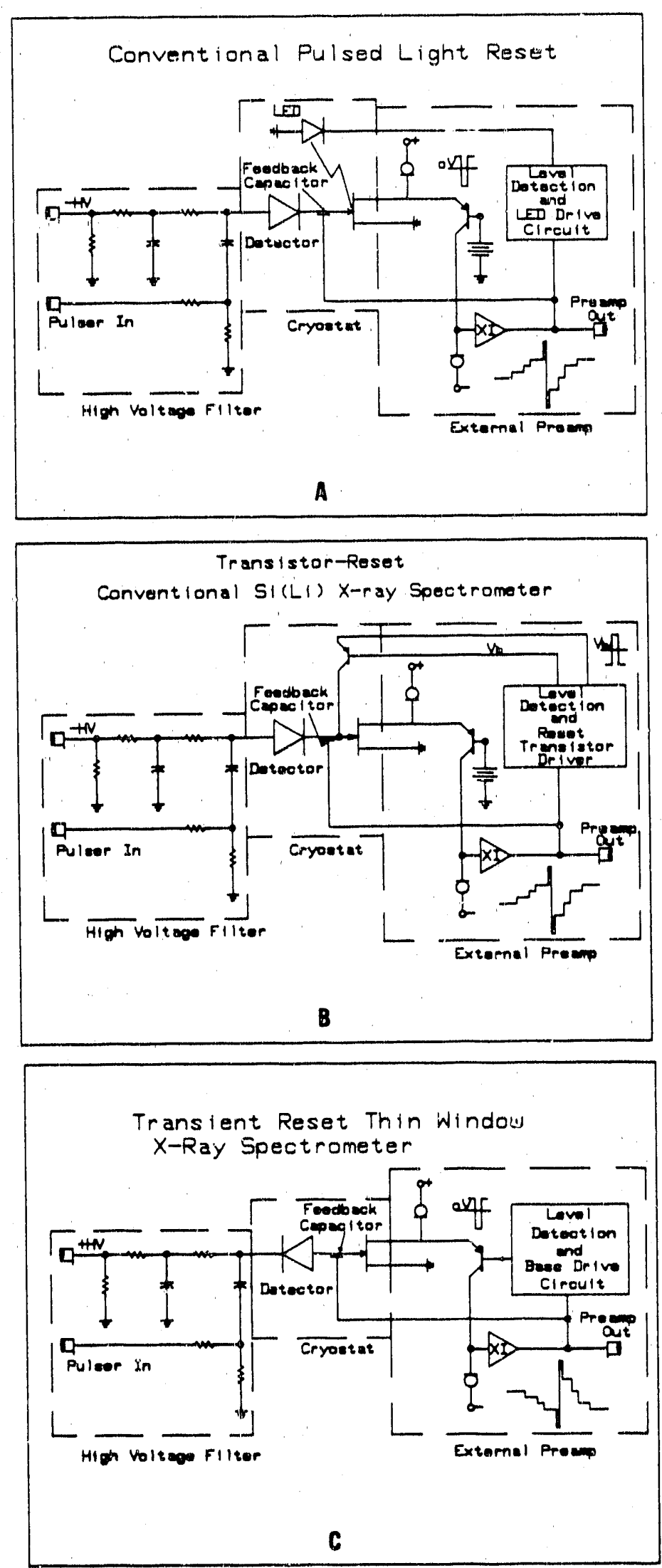

Fig. 3: Simplified schematic of three types of pulsed reset preamplifiers: A) pulse light, B) transistor reset, C) transient reset.
The reset logic module develops a command signal which is adjustable both in amplitude and duration, to pull the base of the folded cascode transistor low. The drain of the FET is driven just sufficiently negative with respect to the gate to cause conduction in the gate-drain diode which restores the charge on the feedback capacitor. The magnitude of the forward bias and the duration of the waveform determine the quantity of charge restored. Charge is injected as the gate-drain diode is driven into conduction by the negative going leading edge of the charge restoration waveform. Most of this charge is extracted on the positive going back edge of this waveform as the gate-drain diode returns to its normal reverse biased condition. A large voltage transient, lasting several tens of microseconds, is observed at the preamplifier output even though the width of the drain waveform is only a few microseconds. This is a serious limitation in applying the technique to high rate applications. A detailed schematic of a tranisient-reset preamplifier with control logic is shown in Fig. 4. A somewhat similar charge restoration method was described in 1970 by Radeka [7]. Here charge restoration was accomplished by applying a high amplitude fast pulse train through the detector capacitance, to cause the gate-to-source diode to conduct. Radeka's method minimizes dead time due to transients after charge restoration.

The new method is limited to removing positive charge from the gate circuit, and since conventional $\mathrm{Si}(\mathrm{Li}) \mathrm{X}$-ray detectors also remove positive charge, it could not be used with such detectors. Justification for the new method lies in the fact that the new technique provides us with the means to measure the noise characteristics of an FET without the necessity of, and therefore the ambiguity introduced by, an operating detector connected to the gate of the FET. To utilize the technique in a practical spectrometer a new detector configuration is needed that permits the gate of the FET to connect to the $\mathrm{P}^{+}$contact of a detector while the $\mathrm{N}^{+}$ contact connects to a positive detector bias supply.

\section{RECONFIGURED-ELECTRODE DETECTOR}

The design of the detector is constrained by the foregoing considerations and by the need to achieve a thick depletion layer to reduce capacitance. At the present time, highpurity silicon with adequate trapping performance for broadenergy-range spectroscopy is not available, so we are forced to use lithium-drifted material. The detector configuration employed here is shown in Fig. 5B. A conventional $3 \mathrm{~mm}$ thick detector, as shown in Fig. 5A, is made by normal procedures. The device is tested with particular emphasis on checking for good lithium compensation by measuring the capacitance vs. bias-voltage relationship. The lithium contact and the gold surface-barrier are then removed by lapping. After the detector is cleaned and etched, the original lithium contact is replaced by an evaporated gold surface- 


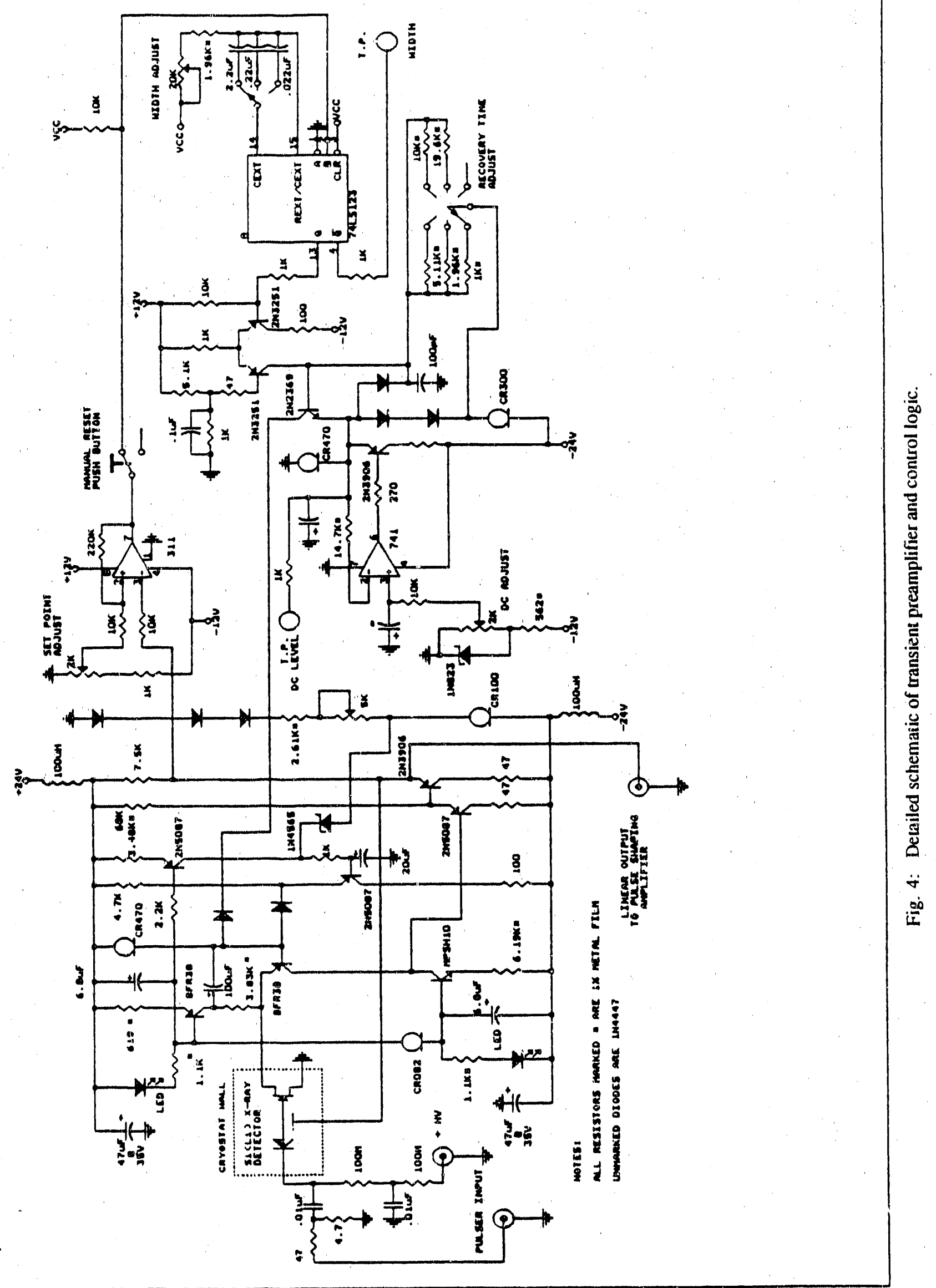




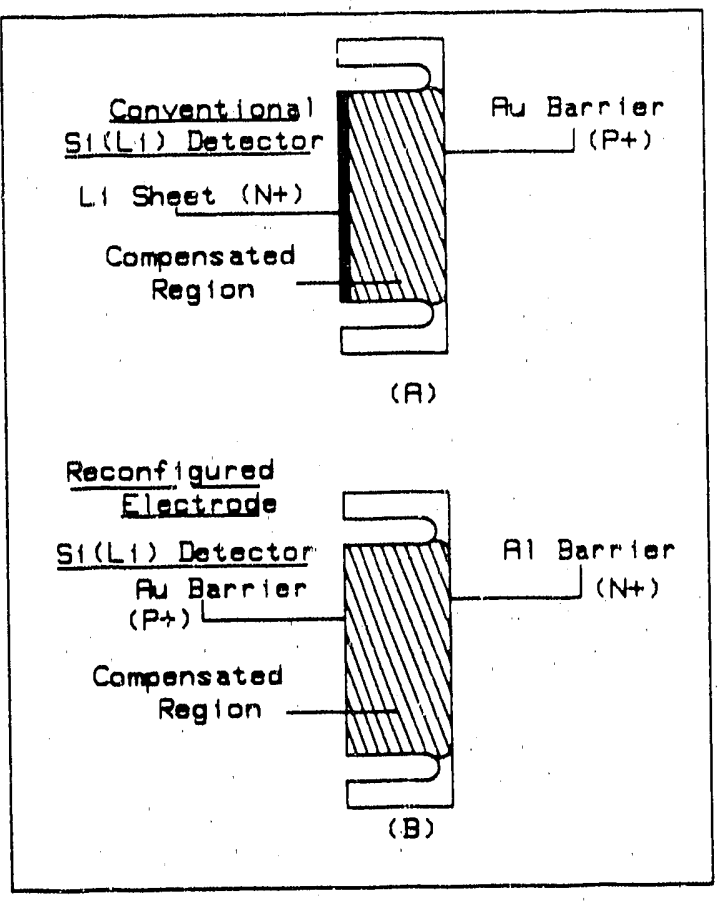

Fig. 5: Cross sectional view of conventional and reconfigured electrode $\mathrm{Si}(\mathrm{Li}) \mathrm{X}$-ray detectors.

barrier. On the other side of the detector, an evaporated aluminum contact is made, replacing the contact that was previously gold. The aluminum contact is an $\mathrm{N}^{+}$barrier to which positive bias is applied. This contact also serves as the entrance window for $\mathrm{X}$-rays. The gold contact, a $\mathrm{P}^{+}$ barrier, is connected to the gate of the input FET. Experience shows that the finished detector is best tested at liquid nitrogen temperatures.

Two important observations have been made using these detectors:

\section{1) Entrance Window Dead Layer}

Using the method discussed in Refs. 8-10, a determination has been made of the thickness of the "dead layer", for the $\mathrm{N}^{+}$window on the seconfigured electrode detector, existing within the silicon itself. Similar determinations have been made for conventional detectors using gold, nickel and implanted boron contacts [10]. The aluminum contact, as seen in Table 1, exhibits a much thinner dead layer than any of the other contacts. One explanation of this relies on the mechanism as discussed in Ref. 9. Charge produced by $X$-ray interactions in the region near the metalsilicon interface is subject to diffusion as well as drift in the electric field. Consequently, some fraction of the carriers will reach the metal-silicon interface and may be lost or reflected depending on the recombination velocity at the interface. It appears likely that the "reflection coefficient" for holes-reaching the aluminum-silicon interface is much higher than that for electrons reaching the gold-

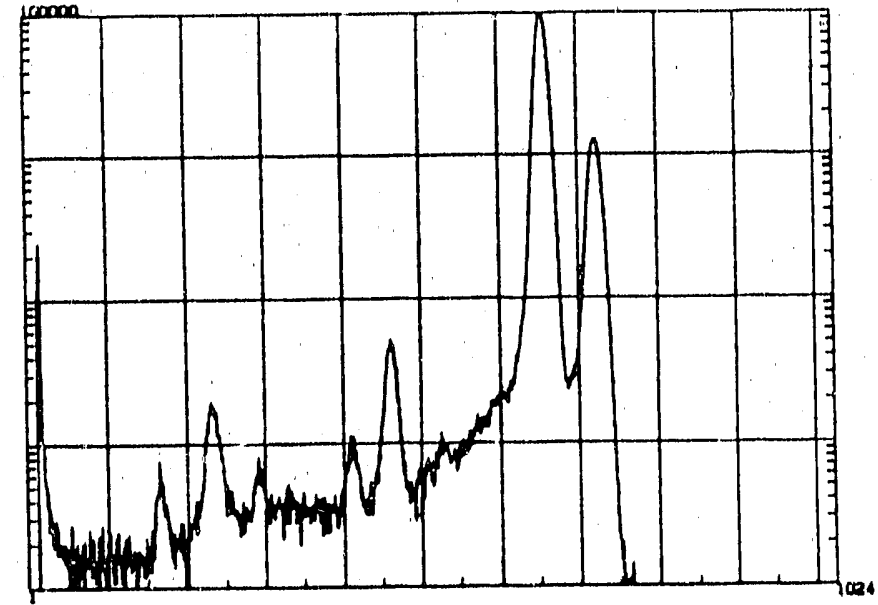

Fig. 6: ${ }^{55} \mathrm{Fe}$ spectrum measured with reconfigured electrode detector and transient reset preamplifier.

\section{TABLE I}

Thickness of the dead layer in the $\mathrm{Si}(\mathrm{Li})$ detector for various contact types.

\begin{tabular}{|l|c|}
\hline Contact Structure & $\begin{array}{c}\text { Measured Si } \\
\text { Dead Layer }(\mu \mathrm{m})\end{array}$ \\
\hline Boron implanted $(p+)$ & 0.46 \\
Gold $(p+)$ & 0.09 \\
Nickel $(p+)$ & 0.08 \\
Aluminum $(n+)$ & $<0.02$ \\
\hline
\end{tabular}

silicon interface of conventional detectors. This can explain the smaller dead layer.

\section{2) Background}

We have also observed a much reduced background at energies below the full energy peak for $\mathrm{X}$-rays such as the $\mathrm{Mn}$ X-rays produced by ${ }^{55} \mathrm{Fe}$ as seen in Fig. 6. In part this may be explained by the thinner silicon dead layer described in the previous section. An additional reason that might be more important is illustrated by the field patterns of Figs. 7 and 8 . The n-type surface channels nearly always present on silicon distort the field lines as shown in these figures. Comparing the conventional detector with a new reconfigured-electrode detector we see that the charge produced near the window of a conventional detector may be collected in surface channels (resulting in partial signals) while that is not the case for the new configuration. This finding is further bolstered by the observation of a 0.10 Fano factor, which we have previously observed only in large volume detectors.

\section{ACKNOWLEDGMENTS}

We would like to acknowledge the many contributions marte by the other members of the Semiconductor Spectrometer Team at Lawrence Berkeley Laboratory including R.H. Pehl, C.P. Cork, D.F. Malone, M.J. Pollard, A. Jue, 


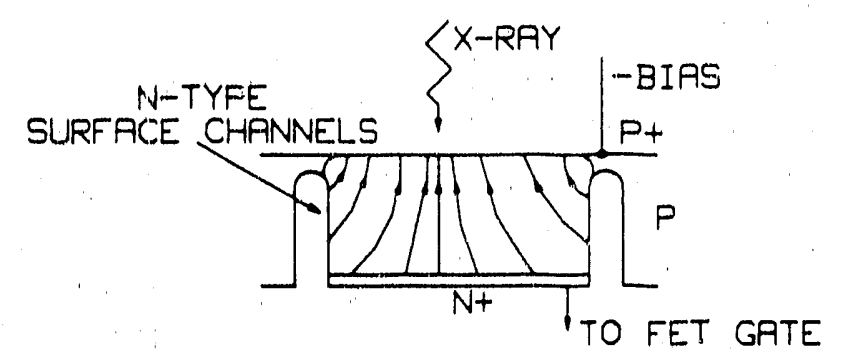

Fig. 7: Field pattern of conventional detector.

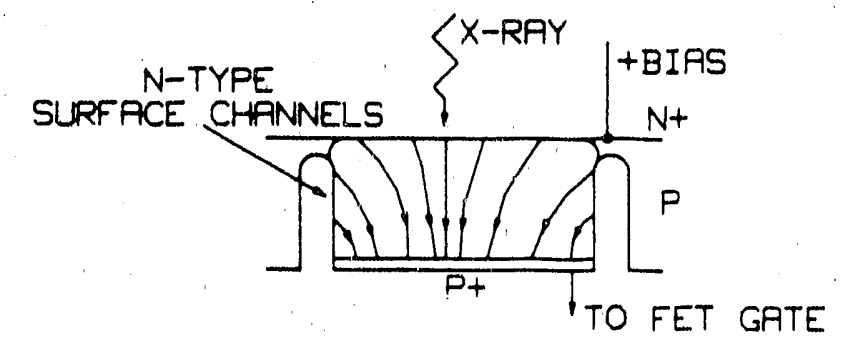

Fig. 8: Field pattern of reconfigured electrode detector.

E. Converse, Y. Wong, H. Sommer and J.T. Walton, in addition to the assistance of R.M. Forment in the manuscript preparation and E. Polonsky for his technical illustrations. A special note of thanks is extended to R.H. Pehl who has asked many insightful questions.
The authors wish to thank the "BBXRT" team at Goddard Space Flight Center for bringing this challenging project to our atsention and for providing partial funding.

This work was supported by the Director's Office of Energy Research, Office of Health and Environmental Research, U.S. Department of Energy under Contract No. DEAC03-76SF00098. Reference to a company or product name does not imply approval or recommendation of the product by the University of California or the U.S. Department of Energy to the exclusion of others that may be suitable.

\section{REFERENCES}

[1] V. Rade,ka, State of the Art of Low Noise Amplifiers for Serniconductor Radiation Detectors, Intl. Symp. on Nuclear Electronics, Versailles, France 46:1-28 (1968')

[2] D. McCammon, private communication (1989).

[3] J. Howes, private communication (1983).

[4] D.A. Landis, F.S. Goulding, R.H. Pehl and J.T. Walton, Pulsed Feedback Techniques for Semiconductor Detector Radiation Spectrometers, IEEE Trans. Nucl. Sci., NS-18. No.1, 115-124 (1.971).

[5] D.A. Landis, C.P. Cork, N.W. Madden and F.S Goulding, Transistor Reset Preamplifier for High Rate, High Resolution Spectroscopy, IEEE Trans. Nucl. Sci., NS-29, No.1, 619-624 (1982).

[6 F.S. Goulding, D.A. Landis and N.W. Madden, Design Philosophy for High Resolution Rate and Throughput Systems, IEEE Trans. Nucl. Sci., NS-30. No.1, 301-310 (1983).

[7] V. Radeka, Charge Amplification Without Charge Leak Resistor, IEEE Trans. Nucl. Sci., NS-17, No.3, $433-439$ (1970).

[8] J. Llacer, E.E. Haller and R.C. Cordi, Entrance Windows in Germanium Low-Energy X-Ray Detectors, IEEE Trans. Nucl Sci., NS-24, No.1, 53-60 (1977).

[9] F.S. Goulding, Some Aspects of Detectors and Electronics for X-Ray Fluorescence Analysis, Nucl. Instr. Meth., 142, 213-223 (1977).

[10] J.M. Jaklevic, J.T. Walton, R.E. McMurray, Jr., N.W. Madden and F.S. Goulding, Semiconductor Detector Performance for Low-Energy X-Rays, Nucl. Instr. Meth., A266 598-601 (1988). 

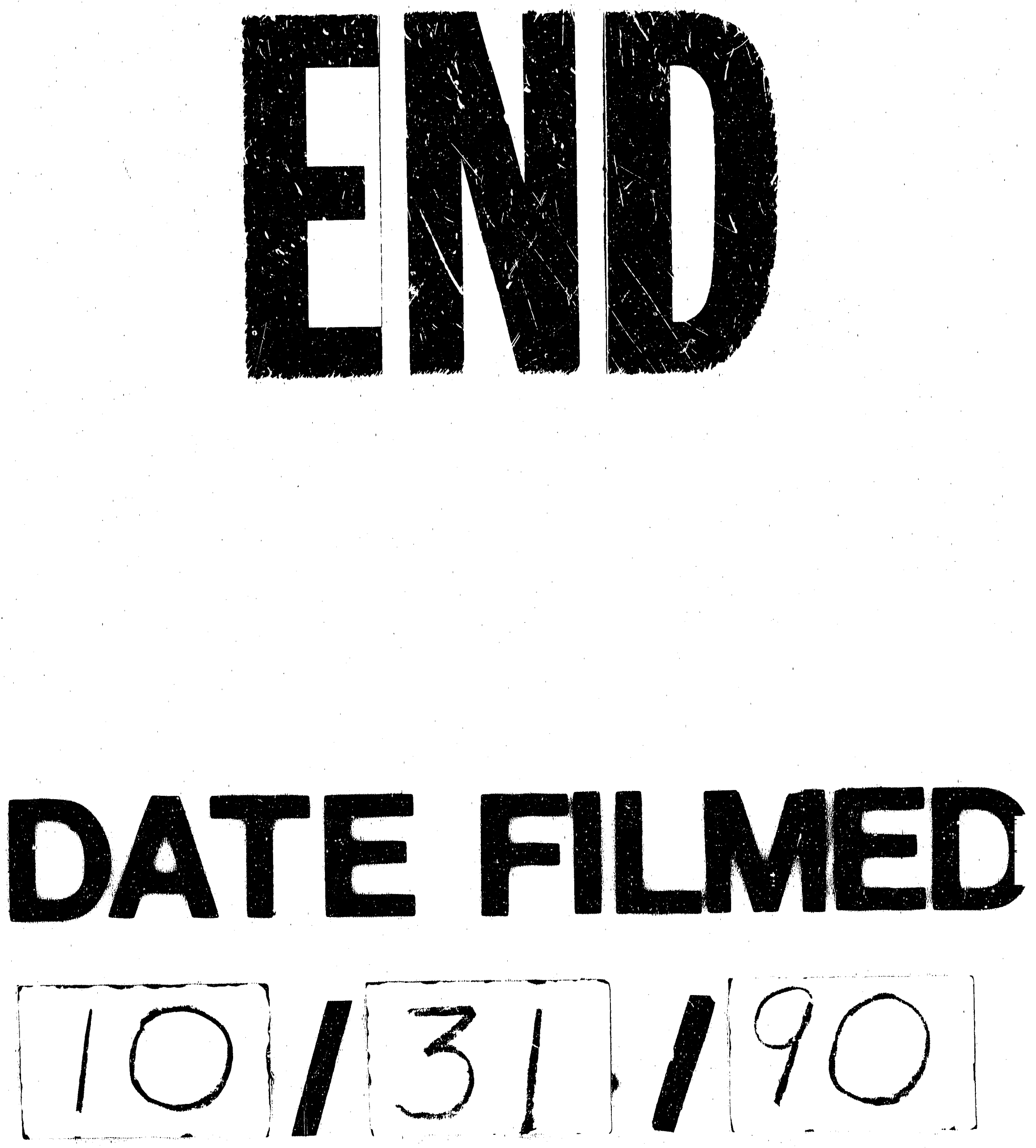
